\title{
The relationship between posttraumatic stress and posttraumatic growth among adolescent and young adult (AYA) cancer patients
}

\author{
Brad Zebrack ${ }^{1}$, Minyoung Kwak ${ }^{2}$, John Salsman ${ }^{3}$, Melissa Cousino ${ }^{4}$, Kathleen Meeske ${ }^{5}$, Christine Aguilar ${ }^{6}$, \\ Leanne Embry ${ }^{6}$, Rebecca Block ${ }^{7}$, Brandon Hayes-Lattin ${ }^{7}$ and Steve Cole ${ }^{8}$ \\ 'University of Michigan School of Social Work, 1080 S. University, Ann Arbor, MI, USA \\ ${ }^{2}$ Gachon University Department of Social Welfare, Seongnam, Korea \\ ${ }^{3}$ Northwestern University Robert Lurie Comprehensive Cancer Center, Chicago, IL, USA \\ ${ }^{4}$ Department of Psychiatry, Boston Children's Hospital/Harvard Medical School, Boston, MA, USA \\ ${ }^{5}$ University of Southern California, Children's Hospital Los Angeles, Los Angeles, CA, USA \\ ${ }^{6}$ University of Texas Health Science Center at San Antonio, San Antonio, TX, USA \\ ${ }^{7}$ Oregon Health Sciences University, Portland, OR, USA \\ ${ }^{8}$ Hopelab Foundation, Redwood City, CA, USA
}

*Correspondence to:

University of Michigan School of Social Work, 1080 S. University, Ann Arbor, Ml 48109-1 106, USA. E-mail: zebrack@umich. edu

Received: 9 December 2013 Revised: 30 April 2014 Accepted: 5 May 2014

\begin{abstract}
Objective: Theories of posttraumatic growth suggest that some degree of distress is necessary to stimulate growth; yet, investigations of the relationship between stress and growth following trauma are mixed. This study aims to understand the relationship between posttraumatic stress symptoms and posttraumatic growth in adolescent and young adult (AYA) cancer patients.

Method: 165 AYA patients aged 14-39 years at diagnosis completed standardized measures of posttraumatic stress and posttraumatic growth at 12 months following diagnosis. Locally weighted scatterplot smoothing and regression were used to examine linear and curvilinear relationships between posttraumatic stress and posttraumatic growth.

Results: No significant relationships between overall posttraumatic stress severity and posttraumatic growth were observed at 12-month follow-up. However, curvilinear relationships between re-experiencing (a posttraumatic stress symptom) and two of five posttraumatic growth indicators (New Possibilities, Personal Strengths) were observed.

Conclusion: Findings suggest that re-experiencing is associated with some aspects of posttraumatic growth but not others. Although re-experiencing is considered a symptom of posttraumatic stress disorder, it also may represent a cognitive process necessary to achieve personal growth for AYAs. Findings call into question the supposed psychopathological nature of re-experiencing and suggest that reexperiencing, as a cognitive process, may be psychologically adaptive. Opportunities to engage family, friends, cancer survivors, or health care professionals in frank discussions about fears, worries, or concerns may help AYAs re-experience cancer in a way that enhances their understanding of what happened to them and contributes to positive adaptation to life after cancer.

Copyright (C) 2014 John Wiley \& Sons, Ltd.
\end{abstract}

\section{Introduction}

Historically, empirical investigations of psychosocial adjustment in adolescents and young adults with cancer (AYAs) have tended to emphasize a psychopathological response that includes mood disorders, anxiety, depression, and posttraumatic stress symptoms [1-5]. Life-threatening medical conditions, such as cancer, can negatively affect AYAs' mental health and potential to achieve developmental tasks [6]. Symptoms of posttraumatic stress are commonly reported among young adult survivors of childhood cancer. For example, Hobbie and colleagues reported that $20.5 \%$ of their childhood cancer survivor population met criteria for lifetime posttraumatic stress disorder [1], and
Kwak et al. reported that $39 \%$ and $44 \%$ of AYAs aged 14-39 years at diagnosis reported moderate to severe levels of posttraumatic stress at 6 and 12 months postdiagnosis, respectively [7]. An emerging body of literature, however, suggests that AYAs are also likely to report and describe ways in which cancer results in greater empathy and concern toward others, increased abilities to cope with tragedy, perceived benefits, personal growth, improved relationships, clarity of future plans, and health competence [8-11]. Barakat and colleagues reported that nearly $85 \%$ of AYA survivors of childhood cancer reported at least one positive outcome from their cancer experience [8].

A diagnosis and treatment of cancer evidently results in both negative and positive consequences for AYA cancer 
patients. Yet, findings to date raise questions about the extent to which positive and negative consequences of cancer are related. Are AYAs who experience positive outcomes different from those who experience and report negative outcomes? Can AYAs experience and report both positive and negative outcomes? Is it possible to cluster or classify AYA cancer patients in such a way that allows us to identify risk factors associated with these outcomes and to predict which AYAs adapt and grow as a result of their experience and which ones suffer and require intensive clinical intervention and support? Answers to these questions will inform the development of psychosocial support interventions that not only minimize the debilitating aspects of cancer but also promote conditions that facilitate AYAs' abilities to cope with cancer and successfully achieve the developmental tasks of adolescence and young adulthood.

In coining the term "posttraumatic growth," Tedeschi and Calhoun [12] theorized that positive outcomes resulting from trauma exposure, such as positive perceptions of oneself, emotional growth, improving relationships with others, and greater appreciation of life are predicated upon individuals appraising their experience as traumatic and distressing enough to shake fundamental life values and world views [13]. This conceptualization suggests that traumatic events may serve as a catalyst for the development of posttraumatic growth because stress facilitates individuals' cognitive process for reconstructing their views of themselves, the world, and the future, which have been challenged by the traumatic events. In this perspective, the experience or perception of trauma exposure as distressing is a necessary but not sufficient precursor to experiencing and reporting growth or benefit. This suggests that perhaps posttraumatic stress and growth are related. Indeed, Barakat, Alderfer, and Kazak found that childhood cancer survivors' reports of posttraumatic stress symptoms were positively correlated with posttraumatic growth [8].

The ability to bear a burden of stress and still report positive outcomes, however, may be finite. Notions of allostatic load suggest that individuals cannot bear a constant bombardment of distress without experiencing detrimental health outcomes [14]. At some point, adversity no longer translates into perceived or experienced benefit; thus, a potentially positive relationship becomes a curvilinear one. Such a curvilinear relationship between posttraumatic stress symptoms (avoidance and intrusive thoughts) and benefit finding have been observed in older adult breast cancer and lymphoma survivors $[15,16]$. Others have reported similar curvilinear relationships between psychological benefits and traumatic exposure in adults of all ages, with perceived benefits being greatest at moderate levels of perceived traumatic distress, but lower at both low and high levels [17].

A final explanation of the relationship between posttraumatic stress and growth may be that the experiences and reports of positive and negative effects are independent. Some individuals exposed to trauma report both positive and negative effects of cancer, others report neither, and still others report low levels of stress and high levels of benefit, or vice versa. Data suggesting no significant relationship between distress and growth have been observed in empirical studies of adult cancer patients, as well [18-20].

Empirical studies of distress and growth among AYAs are few and limited by samples consisting of young people diagnosed as children, who at a young age of diagnosis are limited in their cognitive capacity to recall or derive meaning from their experience or in their ability to compare their psychological state after cancer to what it was before. The purpose of this paper is to examine and interpret the relationship of posttraumatic stress and posttraumatic growth in cancer patients who were diagnosed as adolescents and young adults, when cognitive capacities to evaluate the severity or traumatic nature of cancer is more matured. This information is important, as the development and implementation of comprehensive psychosocial support for AYAs requires attention to risk factors that exacerbate depression, anxiety, or psychological distress but also to factors that promote positive adaptation, coping, resilience, and developmental growth. Understanding the relationship of growth and distress will help advance our understanding of cancer's impact on the lives of AYAs, as well as further the theoretical development and understanding of the impact of trauma on young people's lives.

\section{Method}

\section{Design, procedure, and participants}

A prospective longitudinal study was conducted to examine psychological distress, adaptation, health-related quality of life (HRQOL), and health service utilization over 2 years in AYA patients recently diagnosed with cancer. Baseline data were collected within the first four months of diagnosis and subsequently at 6 and 12 months after the baseline survey. The current study focuses on assessments of posttraumatic stress and posttraumatic growth administered at 12 months following baseline recruitment. Eligibility criteria included patients aged 14-39 years (and anticipated to turn 15 years old during treatment), first diagnosis of any form of invasive cancer, and ability to read and understand English or Spanish. Participating institutions included three pediatric care institutions (Doernbecher Children's Hospital, Portland, OR; Christus Santa Rosa Children's Hospital, San Antonio, TX; Children's Hospital, Los Angeles, CA) and two university-affiliated adult care medical institutions (Oregon Health and Sciences University Hospital, Portland, OR; Cancer Therapy and Research Center, University of Texas, San Antonio, TX). Research staff at each institution monitored 
clinic rosters and subsequently identified and approached a total of 286 eligible patients between March 2008 and April 2010. Fifty-eight patients did not provide consent, either because they refused participation or because physicians denied access to patients who were too sick to participate. An additional 12 AYAs did not return a completed survey after providing consent, and one died. Overall participation rate was $75 \% \quad(n=215)$. Institutional Review Board approval was obtained from each participating site and coordinating center. Informed consent and/or assent was obtained from patients and parents. Additional methodological details are reported elsewhere $[4,21]$.

\section{Measures}

Posttraumatic growth was measured by the Posttraumatic Growth Inventory (PTGI) [12]. Twenty-one items were assessed on a six-point ordinal scale ranging from 0 (no change at all) to 5 (very great change) for five subscales: new possibilities (five items), relating to others (seven items), personal strength (four items), spiritual change (two items), and appreciation of life (three items). Cancer diagnosis was stated in the question stem as the reference for endorsing items. Scores for each of the five subscales were derived by summing response values, and all items were totaled to determine an overall summated PTGI score, ranging from 0 to 105 . Higher scores indicated greater growth. Cronbach's alpha ranged from 0.84 to 0.91 for the five subscales of the PTGI. Cronbach's $\alpha$ was 0.95 for the overall score.

Posttraumatic stress symptoms were measured by the Posttraumatic Stress Diagnostic Scale (PDS) [22]. This measure contains 17 items covering three categories of DSM-IV indicators of posttraumatic stress disorder: (1) re-experiencing (5 items), (2) avoidance (7 items), and (3) arousal (5 items). Participants rated the frequency of each symptom in the past month along a four-point Likert scale ranging from 0 to $3(0=$ not at all or only one time, $1=$ once in a while, $2=$ half of the time, and $3=$ almost always). An overall PDS severity score was calculated by adding responses to items, ranging from 0 to 51 . Subscale items were added to determine severity scores for re-experiencing, avoidance, and arousal. Higher scores indicated greater severity of symptoms. For this sample, Cronbach's $\alpha$ was 0.92 for the overall severity, and 0.87 for re-experiencing, 0.84 for avoidance, and 0.76 for arousal.

Sociodemographic information, including gender, race, relationship/marital status, and age was reported by patients. Age at diagnosis was categorized into three groupings (14-17 years, 18-25, 26-39) to approximate developmental life stages [23]. Clinical data obtained from medical charts included type of cancer and treatment status (on-treatment vs. off-treatment) at survey administration. Surveillance Epidemiology and End Results (SEER) codes were used to categorize cancer type into severity of disease [24]. Three categories of severity of disease were generated: (1) diseases with expected 5-year survival rates greater than $80 \%$ (e.g., testicular cancer, Hodgkin lymphoma); (2) diseases with expected 5-year survival rates between 50-80\% (e.g., osteosarcoma, non-Hodgkin lymphoma); (3) all other invasive malignancies with 5-year survival rates less than $50 \%$ (e.g., leukemias) [25].

\section{Analytic strategy}

Descriptive statistics and independent sample $t$-tests were used to summarize sample characteristics and responses to the PDS and PTGI. Using STATA v.11, results derived from a locally weighted scatterplot smoother (LOWESS) were used to examine the relationship between posttraumatic stress and posttraumatic growth [26]. The locally weighted scatterplot smoother permitted a fitting of nonparametric smoothing curves to the scatterplots without prior assumption of curve shapes (whether relationship would be linear or curvilinear). Based on the results of the locally weighted scatterplot smoother, linear and quadratic regression models were tested. To test for linear and curvilinear associations between posttraumatic stress and posttraumatic growth, PDS severity scores were meancentered and then squared to generate a quadratic term. Regressions of the linear and quadratic terms of the meancentered PDS overall severity score on overall PTGI score was conducted, and included gender, race, relationship status, age, cancer severity, and treatment status as control variables. This procedure was then repeated for each of the individual PDS and PTGI subscales. Bonferroni corrections were applied for multiple comparisons. For PTGI total score and five subscales, level of statistical significance was set at 0.008 to adjust for multiple comparisons.

\section{Results}

\section{Descriptive statistics}

The demographic and clinical characteristics of 165 participants who completed surveys at 12-month follow-up are summarized in Table 1. The overall and subscale scores for PTGI and PDS are displayed in Table 2. With few exceptions, overall PDS and PTGI scores and subscale scores did not vary by gender, race, relationship status, age, severity of disease, and treatment status at 12-month follow-up, after controlling for multiple comparisons $(p<0.008)$ (Table 2).

\section{Relationships between posttraumatic stress and posttraumatic growth}

As seen in Figure 1, the dotted lines indicate the LOWESS fit, and the solid line represents the fit of the quadratic regression to the data. Neither the LOWESS procedure nor regression analyses generated evidence of a statistically 
Table I. Sociodemographic and clinical characteristics of participants $(n=165)$

\begin{tabular}{lc}
\hline Mean age at baseline (SD), years & $\mathbf{2 2 . 8} \mathbf{( 8 . 8 )}$ \\
\hline Gender & \\
Female & $76(46.1 \%)$ \\
Male & $89(53.9 \%)$ \\
Race & \\
White/Caucasian & $74(45.4 \%)$ \\
Hispanic/Latino & $71(43.6 \%)$ \\
Others & $18(11.0 \%)$ \\
Relationship status at I2 months & \\
Married/partnered & $60(37.3 \%)$ \\
Not married/partnered & $101(62.7 \%)$ \\
Age group at diagnosis (baseline) & \\
I3-17 years & $78(47.3 \%)$ \\
I8-25 years & $32(19.4 \%)$ \\
26-39 years & $55(33.3 \%)$ \\
Type of cancer (baseline) & \\
Hodgkin's disease & $19(11.5 \%)$ \\
Non-Hodgkin's lymphoma & $12(7.3 \%)$ \\
Testicular cancer & $8(4.9 \%)$ \\
Female reproductive cancers & $4(2.4 \%)$ \\
Soft-tissue sarcoma & $19(11.5 \%)$ \\
Brain & $13(7.8 \%)$ \\
Leukemia & $43(26.1 \%)$ \\
Other carcinomas & $13(7.9 \%)$ \\
Breast & $13(7.9 \%)$ \\
Bone tumors & $21(12.7 \%)$ \\
Severity of disease & \\
less than 50\% survival rate & $54(32.7 \%)$ \\
$50-80 \%$ survival rate & $67(40.6 \%)$ \\
$80 \%+$ survival rate & $44(26.7 \%)$ \\
Treatment status at I2 months & $85(51.5 \%)$ \\
Stop treatment & $80(48.5 \%)$ \\
On active treatment & \\
\hline &
\end{tabular}

SD, standard deviation.

significant linear or curvilinear relationship between overall PDS and PTGI scores. Scatterplots and multivariate analyses of the Re-experiencing subscale of the PDS and two of the five PTGI subscales, however, suggested significant relationships after controlling for gender, race, relationship status, age, cancer severity, and treatment status. Results from linear and quadratic regression analyses suggest that the relationships between the Reexperiencing subscale of the PDS and two PTGI subscales (New Possibilities, Personal Strength) were statistically significant (Figure 1, Table 3). AYAs' abilities to identify new possibilities and gain personal strength increased significantly as subjects reported higher levels of reexperiencing, but only up to a point, after which PTGI scores decreased as re-experiencing symptoms continued to increase. No other statistically significant relationships between PDS and PTGI subscales were observed after accounting for multiple comparisons and controlling for gender, race, relationship status, age, severity of disease, and treatment status.

\section{Discussion}

This study examined the relationship between the reporting of posttraumatic stress symptoms and posttraumatic growth - the extent to which AYAs perceive their cancer experience as positively influential in their lives. The results indicated that overall PDS and PTGI scores are largely independent of each other and thus consistent with findings in previous studies of cancer patients [16,27-29]. However, a more granular examination of the data, which involved looking at relationships among the respective subscales of these two measures of interest, suggests that some specific aspects of posttraumatic stress may relate in a curvilinear fashion to some specific domains of posttraumatic growth. In terms of new possibilities in life and personal growth, posttraumatic growth appeared related to, and perhaps predicated upon, experiencing some degree of distress associated with exposure to cancer. This interpretation is consistent with the theoretical perspective that one must necessarily perceive a situation or condition as traumatic in order to experience and subsequently report posttraumatic growth [13]. Furthermore, the curvilinear relationship observed here suggests that at some point, symptoms of distress-in this case, re-experiencing-become too burdensome and may impede the cognitive processes necessary for resilience or achievement of posttraumatic growth.

A psychopathological model applied to cancer survivorship suggests that re-experiencing is debilitating, and potentially indicative of a psychiatric disorderposttraumatic stress disorder. However, other theoretical models of adjustment to trauma, and to cancer specifically, suggest that re-experiencing, vis-a-vis intrusive symptoms, intrusive ideation, or rumination, re-introduces memories related to trauma and may be a necessary precursor for establishing meaning, understanding the effect of trauma (i.e., cancer) on one's life, or experiencing personal growth [20,30,31]. Indeed, psychotherapeutic approaches to the treatment of posttraumatic stress disorder involve stimulating patients' memories and reexposing them as a means of developing a cognitive adaptive response. So, hypothesizing the existence of a curvilinear relationship between re-experiencing symptoms and growth supports the assumption that certain levels of stress related to trauma are required to develop enough growth to challenge existing schemes of views on the self and the world. However, if stress symptoms or physical health problems become overwhelming to patients, this may impede potentials for growth or ability to derive meaning from the experience [30].

Psychosocial support interventions are predicated on the need to minimize psychiatric symptoms and negative psychological response to cancer. Yet, comprehensive approaches to psychosocial support require knowledge 
Table 2. Comparison of means (standard deviation) for posttraumatic growth and posttraumatic stress $(n=165)$

\begin{tabular}{|c|c|c|c|c|c|c|c|c|c|c|}
\hline & \multicolumn{6}{|c|}{ Posttraumatic growth (PTGI) score } & \multicolumn{4}{|c|}{ Posttraumatic stress (PDS) severity score } \\
\hline All $(n=165)$ & $67.3(24.3)$ & $23.1(8.8)$ & | $4.2(6.8)$ & | $3.8(4.9)$ & $5.4(3.7)$ & $10.8(4.0)$ & $10.6(9.7)$ & $2.8(3.4)$ & $4.2(4.4)$ & $3.6(3.3)$ \\
\hline \multicolumn{11}{|l|}{ Gender } \\
\hline Female & $69.5(22.9)$ & $24.5(7.7)$ & $14.6(7.0)$ & $14.2(4.9)$ & $5.4(3.7)$ & I I.3 (3.8) & | 1.1 ( $(10.4)$ & $3.1(3.5)$ & $4.3(4.8)$ & $3.7(3.4)$ \\
\hline White & $64.4(24.8)$ & $22.5(9.1)$ & $12.4(6.9)$ & | $3.6(5.2)$ & $4.7(4.0)$ & I0.8 (3.9) & $9.6(9.1)$ & $2.4(3.1)$ & $3.7(4.3)$ & $3.4(2.9)$ \\
\hline Non-white & $69.9(23.4)$ & $23.7(8.5)$ & $15.7(6.4)$ & | $4.1(4.7)$ & $6.0(3.3)$ & $10.8(4.2)$ & $11.4(10.3)$ & $3.1(3.6)$ & $4.5(4.6)$ & $3.9(3.5)$ \\
\hline \multicolumn{11}{|l|}{ Relationship status } \\
\hline No & $65.2(25.1)$ & $22.0(9.3)$ & | 4.3 (6.8) & I $3.7(5.3)$ & 4.9 (3.7) & $10.5(4.3)$ & $10.9(9.9)$ & $2.8(3.5)$ & $4.5(4.7)$ & $3.6(3.1)$ \\
\hline Yes & $71.6(21.6)$ & $25.2(7.2)$ & | $4.2(7.0)$ & | $4.3(4.0)$ & $6.3(3.5)$ & I 1.7 (3.2) & $10.5(9.5)$ & $3.0(3.3)$ & $3.8(4.0)$ & $3.8(3.6)$ \\
\hline \multicolumn{11}{|l|}{ Age } \\
\hline$<50 \%$ survival & $64.5(27.0)$ & $22.0(9.7)$ & $13.9(7.1)$ & I $3.2(5.0)$ & $5.0(3.9)$ & $10.2(4.5)$ & I $0.2(8.9)$ & $2.3(3.1)$ & $4.3(4.1)$ & $3.6(3.3)$ \\
\hline 50-80\% survival & $69.4(22.2)$ & $24.0(7.9)$ & $14.7(6.5)$ & | $4.5(4.4)$ & $5.7(3.7)$ & I $1.4(3.8)$ & $9.5(8.6)$ & $2.6(2.9)$ & $3.7(4.0)$ & $3.4(3.1)$ \\
\hline $80-100 \%$ survival & $67.8(23.4)$ & $23.1(8.9)$ & $13.9(7.0)$ & | $3.7(5.4)$ & $5.4(3.4)$ & I0.8 (3.9) & $12.6(11.8)$ & $3.8(4.1)$ & $4.8(5.3)$ & $4.1(3.6)$ \\
\hline \multicolumn{11}{|l|}{ Treatment status } \\
\hline Off treatment & $69.7(24.7)$ & $23.6(9.2)$ & $15.0(7.0)$ & |4.| (4.9) & $5.8(3.7)$ & I $1.0(4.1)$ & $9.0(9.3)$ & $2.7(3.3)$ & $3.2(4.1)$ & $3.2(3.1)$ \\
\hline On treatment & $65.0(23.3)$ & $22.7(8.3)$ & $13.4(6.6)$ & | 3.5 (4.9) & $5.0(3.6)$ & I $0.7(4.0)$ & I2.3 (9.9) & $3.0(3.5)$ & $5.2(4.5)$ & $4.1(3.4)$ \\
\hline
\end{tabular}

BOLD figures indicate statistically significant difference at $p<0.008$, with Bonferroni correction for multiple comparisons.


Figure I. LOWESS curves demonstrating the relationship between posttraumatic stress and posttraumatic growth

of conditions that not only exacerbate negative outcomes but also promote positive adaptation and coping capability [32,33]. Systematic reviews of posttraumatic growth and its relationship to mental health suggests a potential adaptive significance of posttraumatic growth [19] and that benefit finding may be an outcome of interest in its own right, in that it reflects a positive outcome and not just a mere lack of distress [18]. Promoting posttraumatic growth and/or benefit finding as a cognitive process could potentially induce better coping with the demands of disease and treatment, and thus improve quality of life and adherence to therapy for AYAs, who represent an at-risk population when it comes to adherence [34].
To our knowledge, this study is the first to report on posttraumatic growth outcomes and its relationship to posttraumatic stress in a population of young people diagnosed with cancer in adolescence or young adulthood and contributes to advancing our knowledge about the relationship between posttraumatic stress and growth by using a multi-institutional sample of moderate size. Yet, we acknowledge several limitations of this investigation. First, we acknowledge that the relationship between posttraumatic growth and posttraumatic stress may change beyond 12 months and that additional insights can accrue from examining more distal time points; however, the insights gained from this analysis are highly relevant to understanding relationships between posttraumatic growth 
Table 3. Hierarchical linear regression predicting PTG subscale scores $(n=157)$

\begin{tabular}{|c|c|c|}
\hline & $\begin{array}{c}\text { New possibilities } \\
\beta(p)\end{array}$ & $\begin{array}{c}\text { Personal strength } \\
\beta(p)\end{array}$ \\
\hline Re-experiencing linear term & $0.47(0.04)$ & $0.46(0.007)$ \\
\hline Re-experiencing quadratic term & $-0.10(0.008)$ & $-0.08(0.005)$ \\
\hline Gender ( $($ = male $)$ & $-1.30(0.24)$ & $-0.44(0.58)$ \\
\hline Race (I = non-white) & $3.26(0.005)$ & $0.43(0.60)$ \\
\hline Relationship status ( $\mid=$ yes) & $2.00(0.13)$ & $1.44(0.14)$ \\
\hline Age group $(18-25 \text { yrs })^{a}$ & $-0.77(0.62)$ & $-0.84(0.46)$ \\
\hline Age group $(26-39 \mathrm{yrs})^{\mathrm{a}}$ & $-3.08(0.04)$ & $-1.94(0.08)$ \\
\hline $50 \%-80 \%$ survival $^{\mathrm{b}}$ & $-0.21(0.87)$ & $1.06(0.26)$ \\
\hline $80 \%-100 \%$ survival ${ }^{b}$ & $-0.21(0.89)$ & $0.22(0.84)$ \\
\hline Treatment status ( $\mathrm{I}=$ on treatment) & $-2.02(0.07)$ & $-0.62(0.44)$ \\
\hline$R^{2}$ & 0.152 & 0.100 \\
\hline
\end{tabular}

All figures are unstandardized beta coefficients.

Level of statistical significance set at 0.008 to adjust for multiple comparisons.

aReferent group is "aged 14-17 yrs".

beferent group is " $<50 \%$ survival".

and posttraumatic stress. Second, the statistically significant relationships observed are perhaps muted given the low mean scores and relatively low variability for posttraumatic stress symptom severity, the subsequent low proportion of variance explained by the multivariate models, and the adjustment for multiple comparisons. It is possible that the items operationalizing re-experiencing symptoms of posttraumatic stress are only suggestive of, but not fully capturing the conceptual totality of, rumination-a psychological construct representing both positive and negative cognitive responses. Alternatively, the relationship between posttraumatic stress and growth may indeed be nonexistent, although recent studies report that deliberate rumination predicts posttraumatic growth whereas uncontrolled or intrusive rumination does not $[20,35]$. Including measures that operationalize posttraumatic stress response to cancer as a cognitive process rather than an outcome may better explain why only re-experiencing symptoms are related to posttraumatic growth in AYAs with cancer. We also acknowledge that low mean PDS scores and sample size may have restricted our power to detect significant linear relationships between posttraumatic stress and growth. Unfortunately, we are unable to adequately test sample representativeness by comparing symptom scores observed here to other published studies of posttraumatic stress in young adult cancer survivors due to differences in instrumentation and analytic procedures and the fact that those samples consist of long-term survivors of childhood cancer and not young people diagnosed with cancer as adolescents or young adults $[1,5]$.

Despite limitations, this study provided informative data on the relationship between posttraumatic stress and posttraumatic growth in AYA patients with cancer. Results of the study suggest a need for revisiting re-experiencing solely as an indicator of psychiatric symptomatology and examine its potentially adaptive nature. For example, young cancer survivors worry about their health [36], and worrying about one's health can be considered in pathological terms and necessitating psychological treatment or intervention. However, identification of worry may also be an opportunity for engagement and reframing around promoting positive health behaviors, adhering to therapy, maintaining posttreatment surveillance and long-term follow-up. Opportunities to engage family, friends, other young adult cancer survivors, or health care professionals in frank discussions about their fears, worries, or concerns may help AYAs reexperience cancer in a way that enhances their understanding of what happened to them and contributes to personal growth and positive adaptation to life after cancer. Future research is needed to examine the efficacy of cognitivebehavioral and exposure-based therapies that utilize reexperiencing as a process by which growth after trauma is facilitated. In addition, future investigation of peer support programs aimed at promoting positive growth postcancer is needed. Lastly, it will be important to further investigate and identify the threshold by which distress symptoms become too great and potentials for growth and meaningmaking are exceeded.

\section{Acknowledgement}

This study supported by HopeLab Foundation, Redwood City, CA.

\section{References}

1. Hobbie WL, Stuber M, Meeske KA, et al. Symptoms of posttraumatic stress in young adult survivors of childhood cancer. $J$ Clin Oncol 2000;18(24):4060-4066.

2. Jorngarden A, Mattsson E, Essen LV. Healthrelated quality of life, anxiety and depression among adolescents and young adults with cancer: A prospective longitudinal study. Eur J Cancer 2007;43:1952-1958.

3. Meeske KA, Ruccione K, Globe DR, et al. Posttraumatic stress, quality of life, and psychological distress in young adult survivors of childhood cancer. Oncol Nurs Forum 2001;28(3):481-489.

4. Kwak M, Zebrack B, Meeske KA, et al. Trajectories of psychological distress in adolescent and young adult cancer patients: A one-year longitudinal study. J Clin Oncol 2013;31(17):2160-2166.

5. Stuber ML, Meeske KA, Krull KR, et al. Prevalence and Predictors of Posttraumatic Stress Disorder in Adult Survivors of Childhood Cancer. Pediatrics 2010;125(5): e1124-e1134.

6. Zebrack B, Hamilton R, WilderSmith A. Psychosocial outcomes and service use among young adults with cancer. Semin Oncol 2009; 36(5):468-477.

7. Kwak M, Zebrack B, Meeske KA, et al. Prevalence and predictors of post-traumatic stress symptoms in adolescent and young adult cancer survivors: A 1-year follow-up study. Psycho-Oncology 2013;22(8):1798-1806.

8. Barakat LP, Alderfer MA, Kazak AE. Posttraumatic growth in adolescent survivors of cancer and their mothers and fathers. $J$ Pediatr Psychol 2006;31(4):413-419.

9. Parry C, Chesler M. Thematic evidence of psychosocial thriving in survivors of childhood cancer. Qual Health Res 2005;15(8):1055-1073. 
10. Bellizzi KM, Smith A, Schmidt S, et al. Positive and negative psychosocial impact of being diagnosed with cancer as an adolescent or young adult. Cancer 2012;118(20):51555162 .

11. Phipps S, Long AM, Ogden J. Benefit finding scale for children: Preliminary findings from a childhood cancer population. J Pediatr Psychol 2007:32(10):1264-1271.

12. Tedeschi RG, Calhoun LG. The Posttraumatic Growth Inventory: Measuring the Positive Legacy of Trauma. J Trauma Stress 1996;9(3): 455-471.

13. Tedeschi RG, Calhoun LG. Posttraumatic growth: Conceptual foundations and empirical evidence. Psychol Inq 2004;15(1):1-18.

14. McEwen BS, Stellar E. Stress and the Individual: Mechanisms Leading to Disease. Arch Intern Med 1993;153:2093-2101.

15. Lechner SC, Carver CS, Antoni MH, et al. Curvilinear associations between benefit findings and psychosocial adjustment to cancer. $J$ Consult Clin Psychol 2006;74(5):828-840.

16. Smith SK, Samsa G, Ganz PA, et al. Is there a relationship between posttraumatic stress and growth after a lymphoma diagnosis? PsychoOncology 2014;23(3):315-321.

17. Linley P, Joseph S. Positive change following trauma and adversity: A review. J Trauma Stress 2004;17(1):11-21.

18. Helgeson VS, Reynolds KA, Tomich PL. A metaanalytic review of benefit-finding and growth. J Consult Clin Psychol 2006;74(5):797-816.

19. Barskova T, Oesterreich R. Post-traumatic growth in people living with a serious medical condition and its relations to physical and mental health: A systematic review. Disabil Rehabil 2009;31(21):1709-1733.

20. Morris BA, Shakespeare-Finch J. Rumination, post-traumatic growth, and distress: structural equation modelling with cancer survivors. Psycho-Oncology 2011;20:1176-1183.

21. Zebrack B, Block R, Hayes-Lattin B, et al. Psychosocial service use and unmet need among recently diagnosed adolescent and young adult cancer patients. Cancer 2013;119:201-214.

22. Foa EB. Posttraumatic Stress Diagnostic Scale: Manual. National Computer Systems: Minneapolis, MN, 1995.

23. Arnett JJ. Emerging adulthood: A theory of development from the late teens through the twenties. Am Psychol 2000;55(5):469-480.

24. Howlader N, Noone AM, Krapcho M, et al. SEER Cancer Statistics Review, 1975-2008. 2011. (Available from: http://seer.cancer.gov/ csr/1975_2008/). Accessed 20 May 2014.

25. Bleyer A. Latest estimates of survival rates of the 24 most common cancers in adolescent and young adult Americans. J Adolesc Young Adult Oncol 2011;1(1):37-41.

26. Cleveland WS. Robust locally weighted regression and smoothing scatterplots. $J \mathrm{Am}$ Stat Assoc 1979;74:829-836.

27. Salsman JM, Segerstrom SC, Brechting EH, et al. Posttraumatic growth and PTSD symptomatology among colorectal cancer survivors: a 3-month longitudinal examination of cognitive processing. Psycho-Oncology 2009;18:30-41.

28. Widows MR, Jacobsen PB, Booth-Jones M, et al. Predictors of posttraumatic growth following bone marrow transplantation for cancer. Health Psychol 2005;24(3): 266-273.

29. Cordova MJ, Giese-Davis J, Golant M, et al. Breast cancer as trauma: Posttraumatic stress and posttraumatic growth. J Clin Psychol Med Settings 2007;14:308-319.

30. Tomich P, Helgeson VS. Is finding something good in the bad always good? Benefit finding among women with breast cancer. Health Psychol 2004;23(1):16-23.

31. Creamer M, Burgess P, Pattison P. Reaction to trauma: A cognitive processing model. J Abnorm Psychol 1992;101(3):452-459.

32. Zebrack B, Zeltzer L. Editorial: Living beyond the Sword of Damocles: Surviving childhood cancer. Expert Rev Anticancer Ther 2001;1(2):163-164.

33. Haase JE. The adolescent resilience model as a guide to interventions. J Pediatr Oncol Nurs 2004;21(5):289-299.

34. Butow P, Palmer S, Pai A, et al. Review of adherence-related issues in adolescents and young adults with cancer. J Clin Oncol 2010;28(32):4800-4809.

35. Chan MWC, Ho SMY, Tedeschi RG, et al. The valence of attentional bias and cancer-related rumination in posttraumatic stress and posttraumatic growth among women with breast cancer. Psycho-Oncology 2011;20:544-552.

36. Zebrack BJ, Donohue JE, Gurney JG, et al. Psychometric evaluation of the Impact of Cancer (IOC-CS) scale for young adult survivors of childhood cancer. Qual Life Res 2010;19(2):207-218. 